\title{
Chiari 1.5 malformation, accessory odontoid synchondrosis, and ventral compression: case report
}

\author{
Dany Hage ${ }^{1}$, Joe Iwanaga ${ }^{1,2}$, CJ Bui ${ }^{4}$, Aaron S. Dumont ${ }^{1}$, R. Shane Tubbs ${ }^{1,2,3,4,5}$ \\ ${ }^{1}$ Department of Neurosurgery, Tulane Center for Clinical Neurosciences, Tulane University School of Medicine, New Orleans, LA, ${ }^{2}$ Department of \\ Neurology, Tulane Center for Clinical Neurosciences, Tulane University School of Medicine, New Orleans, LA, ${ }^{3}$ Department of Structural \& Cellular \\ Biology, Tulane University School of Medicine, New Orleans, LA, ${ }^{4}$ Department of Neurosurgery and Ochsner Neuroscience Institute, Ochsner Health \\ System, New Orleans, LA, USA, ${ }^{5}$ Department of Anatomical Sciences, St. George’s University, St. George’s, Grenada, West Indies
}

\begin{abstract}
The pathogenesis of Chiari 1 malformations has been explained in several different ways, but extensive evidence suggests a relationship between loss of volume within the posterior cranial fossa and Chiari 1 presentations. It is important to be able to differentiate Chiari 1.5 from Chiari 1 malformations as they have similar clinical presentations, but the latter have progressed further and are characterized by caudal herniations of the brain stem through the foramen magnum. Despite the similarities of presentation, Chiari 1.5 malformations have greater rates of complications following posterior decompression surgeries, which are typically performed to relieve ventral compression. An improved understanding of the odontoid synchondroses could lead to better understanding of Chiari malformations and lead to improved treatment of patients with these presentations. Here we present a rare case of an accessory odontoid synchondrosis in a patient with a Chiari 1.5 malformation and ventral compression.
\end{abstract}

Key words: Dens, Odontoid process, Anatomy, Chiari malformations, Odontoid synchondroses

Received October 7, 2020; Revised October 28, 2020; Accepted October 29, 2020

\section{Introduction}

The odontoid synchondroses are cartilaginous articulations separating the odontoid process from the body of the axis (Fig. 1) [1]. They normally become fully fused between the ages of five and seven years, usually forming a line on radiographs until 11 years; so most cases involving fracture of the synchondrosis are in children [1-3]. In this report, we present a rare case of an accessory odontoid synchondrosis in a patient with a Chiari 1.5 malformation and ventral com-

\footnotetext{
Corresponding author:

Joe Iwanaga (iD

Department of Neurosurgery, Tulane Center for Clinical Neurosciences, Tulane University School of Medicine, New Orleans, LA 70112, USA E-mail: iwanagajoeca@gmail.com
}

pression.

\section{Case Report}

A 13-year-old boy presented with diplopia and occasional headaches. The symptoms were ongoing for three months and were more pronounced in upon wakening in the morning. There was no past surgical or medical history. On examination, a left abducens nerve palsy was identified and his gag reflex was absent. The remainder of his motor and sensory exams were normal as was his cerebellar and deep tendon reflex exams. Magnetic resonance imaging (MRI) revealed a Chiari 1.5 malformation without syringomyelia (Fig. 2). Additionally, ventral compression $(8 \mathrm{~mm})$ of the anterior brainstem was noted. There was no hydrocephalus. An additional synchondrosis of the odontoid process, superior to the normal subdental synchondrosis, was identified (Fig. 2). This synchondrosis 


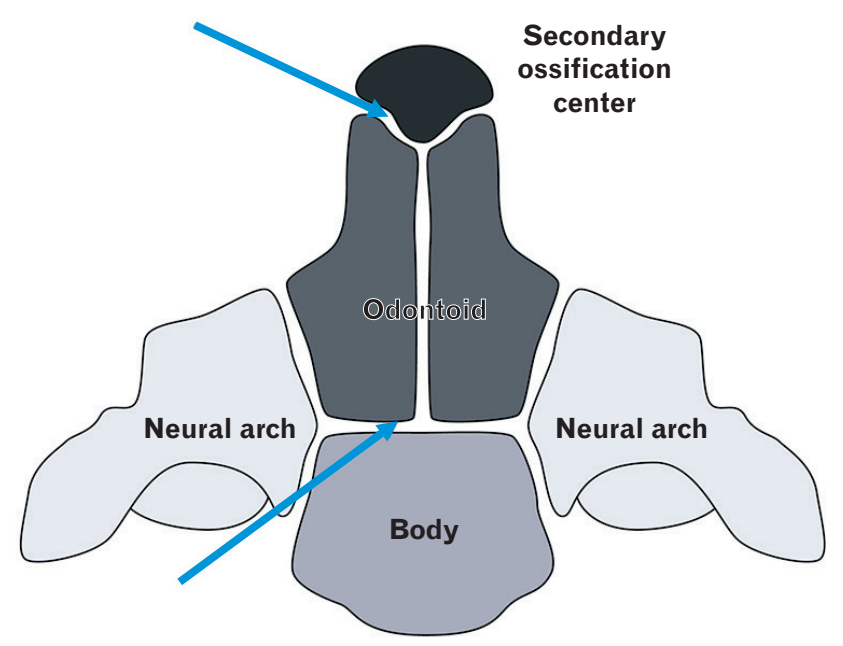

Fig. 1. Schematic drawing of the ossification centers and synchondroses (arrows) of the C2 vertebra.

was located at the midportion of the odontoid process inferior to where the apical synchondrosis would have been located and gave the odontoid process and elongated appearance. A posterior fossa decompression procedure was offered but was declined by the family.

\section{Discussion}

The odontoid synchondroses are present at birth and serve as cartilaginous separations between the odontoid process, neural arches and body of the axis [1]. They are categorized as dentocentral (subdental), between the odontoid process and the body of the dens, neurocentral, between the odontoid process and the neural arches, and apical just below the apex of the odontoid process $[1,2,4]$. Typically, the odontoid process has fused fully with the body and the neural arches by 5 to 7 years of age, leaving adults with a dentocentral remnant within the cancellous bone that can be visualized on MRI as a hypointense ring between the superior surface of the body of $\mathrm{C} 2$ and the inferior surface of the odontoid process [1-3]. The apical synchondrosis fuses by ten years [3]. Owing to these fusion patterns, fractures of the odontoid synchondroses usually affect children under the age of 10 years.

Our case shows no sign of traumatic injury, but it displays a Chiari 1.5 malformation. Chiari 1.5 malformations exhibit similar tonsillar herniations to Chiari 1 malformations, but they are distinguished by caudal brainstem herniations through the foramen magnum [5-8]. The diagnosis of Chiari

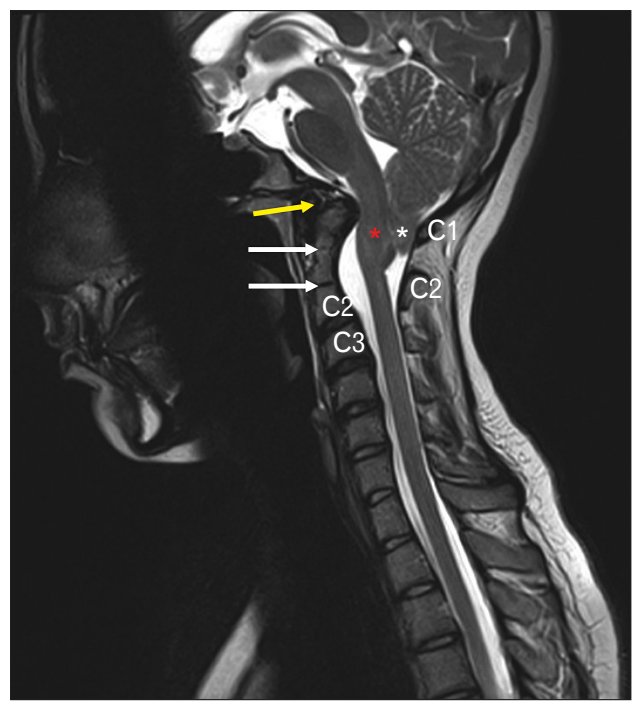

Fig. 2. Two odontoid synchondroses in the same C2 vertebra (white arrows) shown in a midsagittal magnetic resonance imaging image. The upper white arrow notes the accessory synchondrosis. Note the cerebellar tonsils (asterisk), and the lower part of the brainstem (red star) located below the foramen magnum i.e., Chiari 1.5 malformation. The anterior arch of the atlas (yellow arrow) is not ossified. The posterior arch of $\mathrm{C} 1$ and spinous process of $\mathrm{C} 2$ are also shown.

1.5 malformation has posed challenges because of the similarity in clinical presentation to Chiari 1 malformation, including (but not limited to) dyspnea, jaw pain, drop attacks, dysarthria, opisthotonos, perioral numbness, negative gag reflex, lethargy, headaches, and limb weakness [6-8]. Despite the similarity in presentations, it is important to identify Chiari 1.5 malformations early and differentiate them from Chiari 1 malformations as their operative outcomes can differ $[5,6]$. In a study of 130 pediatric patients with Chiari malformations, Tubbs et al. noted a difference in the incidence of unresolved or recurring episodes of syringomyelia following posterior fossa decompression between patients presenting with Chiari 1 (6.8\%) and Chiari 1.5 (13.6\%) malformations [6, 7, 9].

Many different explanations of the pathogenesis of Chiari malformations have been proposed, but because a wide variety of anomalies can arise, no definitive account has yet been agreed [10]. Nevertheless, extensive evidence demonstrates that reduction of the posterior cranial fossa volume contributes to hindbrain herniation and the development of a Chiari 1 malformation in many cases $[11,12]$. Such decreases in volume have been associated with developmental abnormalities disrupting the rotation of the tentorium cerebelli and petrous bone during the embryonic, fetal, and postnatal periods, leading to an overcrowded posterior cranial fossa [11]. 
Although ventral compression can be seen in patients with Chiari 1.5 malformation, commonly associated vertebral variations include atlanto-occipital fusion and Klippel-Feil syndrome and not an accessory synchondrosis of the odontoid process as seen in the present case (Fig. 2).

Furthermore, there is a high incidence of craniovertebral junction (CVJ) abnormalities among the Chiari 1 malformation population. Many hypotheses have linked the development and migration of the odontoid process, axis, and clivus to shrinkage of the posterior cranial fossa, exacerbating the conditions and causing further tonsillar herniation $[5,13]$. Studies of pediatric populations have revealed a correlation between retroflexion of the odontoid process and the presence of a Chiari 1 malformation or other skeletal disorder with CVJ abnormalities $[1,14,15]$. In fact, excessive retroflexion of the odontoid process has been classed as a causative agent of ventral compression among Chiari 1 patients and this was seen in the present case [15]. Currently, it is standard protocol to perform a posterior decompression on Chiari 1 patients exhibiting ventral compression [15]. In view of the differing surgical outcomes between Chiari 1 and 1.5 patients, it is essential for physicians to distinguish between them and consider their variable anatomy $[6,7,9,15]$.

Although variations and pathology such as atlanto-occipital fusion and Klippel-Feil syndrome have been reported in a patient with a Chiari 1.5, to our knowledge, an accessory synchondrosis of the odontoid process with a Chiari 1.5 malformation has not been described in the literature. Owing to the lack of available information, the clinical implications of a duplicated odontoid synchondrosis are still unclear, and it is difficult to be certain that a concomitant Chiari malformation contributes to its development. Elucidating this mechanism could give greater insight into the pathogenesis of Chiari malformations and provide us with a more comprehensive plan for diagnosing and treating patients with these presentations. Since there are differences between the surgical outcomes of Chiari 1 and 1.5 malformation patients, differentiating the two is imperative for ensuring the greatest patient benefit.

\section{ORCID}

Dany Hage: https://orcid.org/0000-0002-2404-3449

Joe Iwanaga: https://orcid.org/0000-0002-8502-7952

CJ Bui: https://orcid.org/0000-0001-5760-1382

Aaron S. Dumont: https://orcid.org/0000-0002-8077-8992
R. Shane Tubbs: https://orcid.org/0000-0003-1317-1047

\section{Author Contributions}

Conceptualization: JI, RST. Data acquisition: ASD, RST. Data analysis or interpretation: DH, CJB. Drafting of the manuscript: DH, JI. Critical revision of the manuscript: ASD, RST. Approval of the final version of the manuscript: all authors.

\section{Conflicts of Interest}

No potential conflict of interest relevant to this article was reported.

\section{References}

1. Akobo S, Rizk E, Loukas M, Chapman JR, Oskouian RJ, Tubbs RS. The odontoid process: a comprehensive review of its anatomy, embryology, and variations. Childs Nerv Syst 2015;31:202534 .

2. Gebauer M, Lohse C, Barvencik F, Pogoda P, Rueger JM, Püschel K, Amling M. Subdental synchondrosis and anatomy of the axis in aging: a histomorphometric study on 30 autopsy cases. Eur Spine J 2006;15:292-8.

3. Muthukumar N. Odontoid synchondrosis fracture treated by c1-2 polyaxial screw-rod fixation. J Korean Neurosurg Soc 2014;55:212-4.

4. Cramer GD, Darby SA. Clinical anatomy of the spine, spinal cord, and ANS. 3rd ed. St. Louis: Mosby; 2013. 688 p.

5. Kim IK, Wang KC, Kim IO, Cho BK. Chiari 1.5 malformation: an advanced form of Chiari I malformation. J Korean Neurosurg Soc 2010;48:375-9.

6. Azahraa Haddad F, Qaisi I, Joudeh N, Dajani H, Jumah F, Elmashala A, Adeeb N, Chern JJ, Tubbs RS. The newer classifications of the chiari malformations with clarifications: an anatomical review. Clin Anat 2018;31:314-22.

7. Tubbs RS, Iskandar BJ, Bartolucci AA, Oakes WJ. A critical analysis of the Chiari 1.5 malformation. J Neurosurg 2004;101(2 Suppl):179-83.

8. Capra V, De Marco P, Merello E, Baffico AM, Baldi M, Divizia MT, Gimelli S, Mallet D, Raso A, Mascelli S, Tomà P, Rossi A, Pavanello M, Cama A, Magnani C. Craniosynostosis, hydrocephalus, Chiari I malformation and radioulnar synostosis: probably a new syndrome. Eur J Med Genet 2009;52:17-22.

9. Tubbs RS, McGirt MJ, Oakes WJ. Surgical experience in 130 pediatric patients with Chiari I malformations. J Neurosurg 2003;99:291-6.

10. Shoja MM, Johal J, Oakes WJ, Tubbs RS. Embryology and pathophysiology of the Chiari I and II malformations: a com- 
prehensive review. Clin Anat 2018;31:202-15.

11. Shoja MM, Ramdhan R, Jensen CJ, Chern JJ, Oakes WJ, Tubbs RS. Embryology of the craniocervical junction and posterior cranial fossa, part II: embryogenesis of the hindbrain. Clin Anat 2018;31:488-500.

12. Shoja MM, Ramdhan R, Jensen CJ, Chern JJ, Oakes WJ, Tubbs RS. Embryology of the craniocervical junction and posterior cranial fossa, part I: development of the upper vertebrae and skull. Clin Anat 2018;31:466-87.
13. Goel A. Basilar invagination, Chiari malformation, syringomyelia: a review. Neurol India 2009;57:235-46.

14. Cesmebasi A, Loukas M, Hogan E, Kralovic S, Tubbs RS, Cohen-Gadol AA. The Chiari malformations: a review with emphasis on anatomical traits. Clin Anat 2015;28:184-94.

15. Besachio DA, Khaleel Z, Shah LM. Odontoid process inclination in normal adults and in an adult population with Chiari malformation Type I. J Neurosurg Spine 2015;23:701-6. 